\title{
Positive association between blood natural killer $T$ cells and liver enzymes ALT, AST and GGT levels
}

Soledad López ${ }^{1,2}$, David Pozo ${ }^{1,2}$ Sara Garcia-Serrano3,4, Carolina Gutierrez-Repiso ${ }^{3}$, Francisca Rodriguez-Pacheco ${ }^{3}$, Ailec Ho-Plagaro ${ }^{3}$, Miguel A Alaminos-Castillo3, Sergio Valdes ${ }^{3}$, Montserrat Gonzalo3, Raul J. Andrade 5 , Eduardo Garcia-Fuentes ${ }^{5,6}$

1Department of Medical Biochemistry, Molecular Biology and Immunology, The University of Seville Medical School, Seville, Spain. 2 CABIMER-Andalusian Center for Molecular

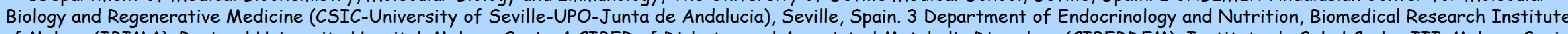

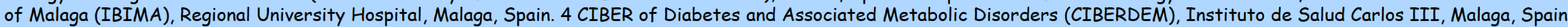

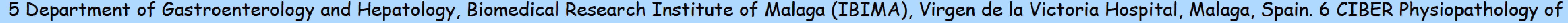
Obesity and Nutrition (CIBERObn), Instituto Salud Carlos III, Malaga, Spain

\section{Introduction}

Obesity is associated to a pro-inflammatory state with a different pattern of response from the classical response. Also, obesity is associated to non-alcoholic fatty liver disease (NAFLD). In this sense, natural killer T (NKT) cells are a subset of innate immune cells that abundantly reside within the liver and are readily activated by lipid antigens. However, the phenotype and functional characteristics of these cells are no clear in the immune homeostasis in obesity.

\section{Desing \& Methods}

Peripheral blood mononuclear cell (PBMC) from 17 lean controls and 20 morbidly obese (MO) patients with normal levels of liver enzymes ALT, AST and GGT were isolated to address the association between iNKT cells and ALT, AST and GGT. PBMC were analyzed by FACS CANTO II flow cytometry.

\section{Results}

In PBMC, no differences were observed in the frequency of NKT cells of $M O$ and lean subjects. However, into MO group, we found a significant correlation between NKT cells and ALT levels $(r=0.611, p=0.015)$ (Figure 1).

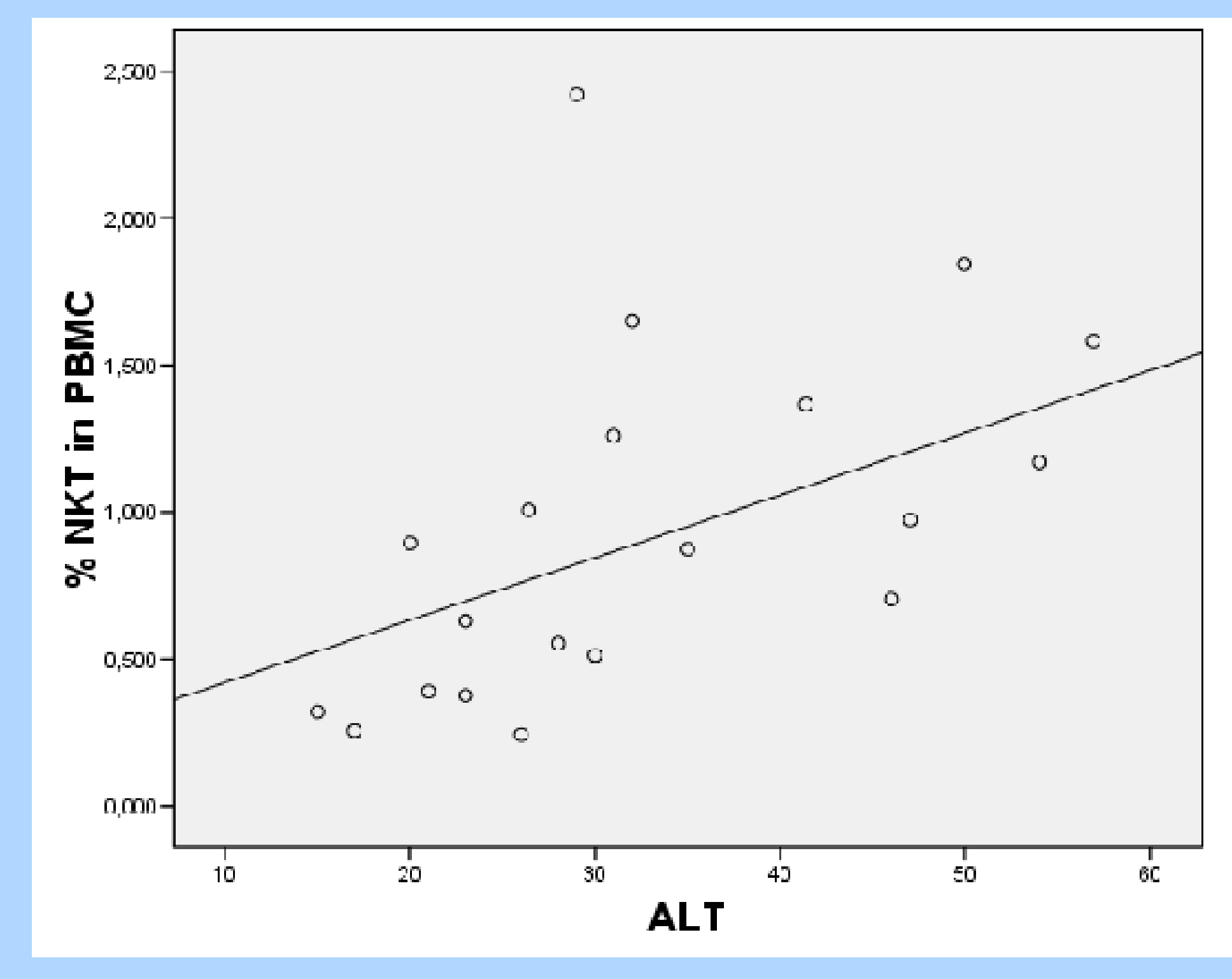

Figure 1. Correlation between NKT cells and ALT levels.
No differences were observed in CD4+ and CD8+iNKT cells in PBMC from $M O$ compared with lean subjects, but a positive correlation between CD4+iNKT cells with ALT $(r=0.476, p=0.043)$ and $G G T$ $(r=0.581, p=0.029)$ was found (Figure 2).
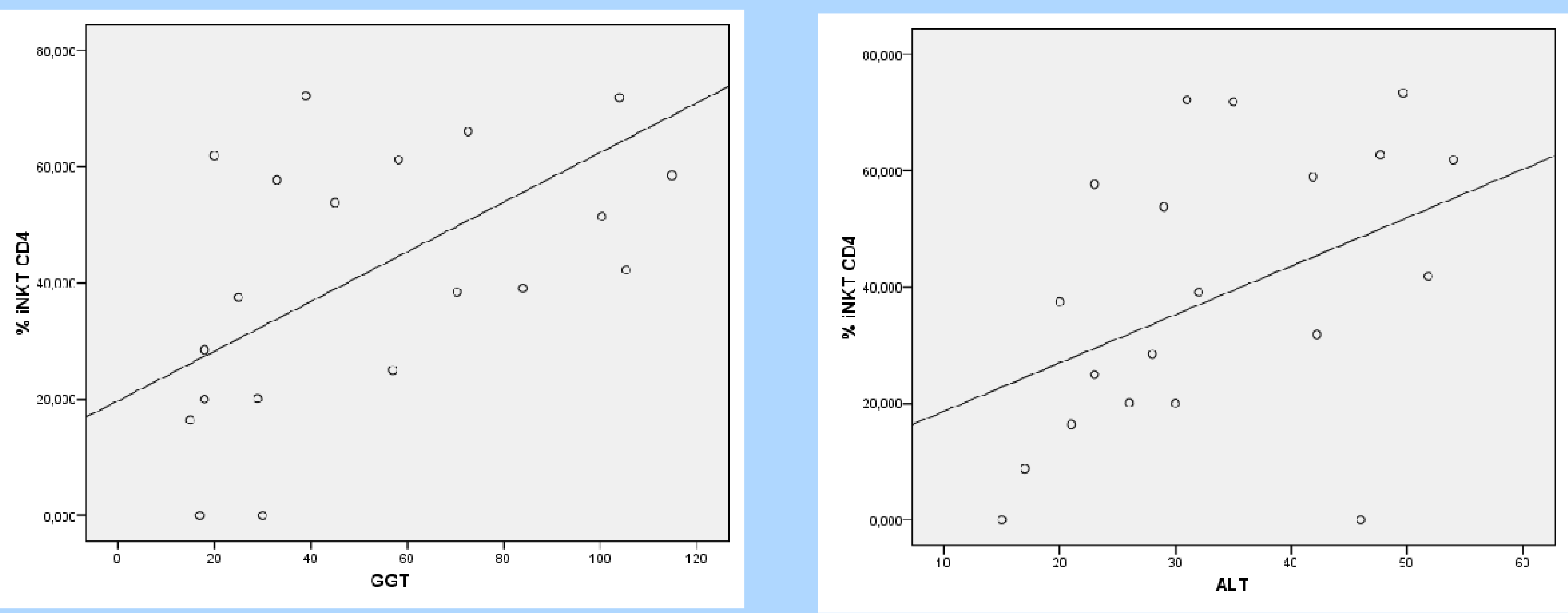

Figure 2. Correlation between CD4+iNKT cells with GGT and ALT levels.
CD56+NKT and CD56+iNKT cells were increased in PBMC of MO subjects $(p<0.001)$ (Figure 3$)$. The frequency of CD69+CD25+ iNKT cells (early and later activated iNKT) was significantly increased in PBMC from MO subjects ( $p<0.001$ ) (Figure 3 ). These activated iNKT cells presented a significantly and positive correlation with AST $(r=0.677, p=0.006)$ and ALT $(r=0.693, p=0.004)$ (Figure 4).
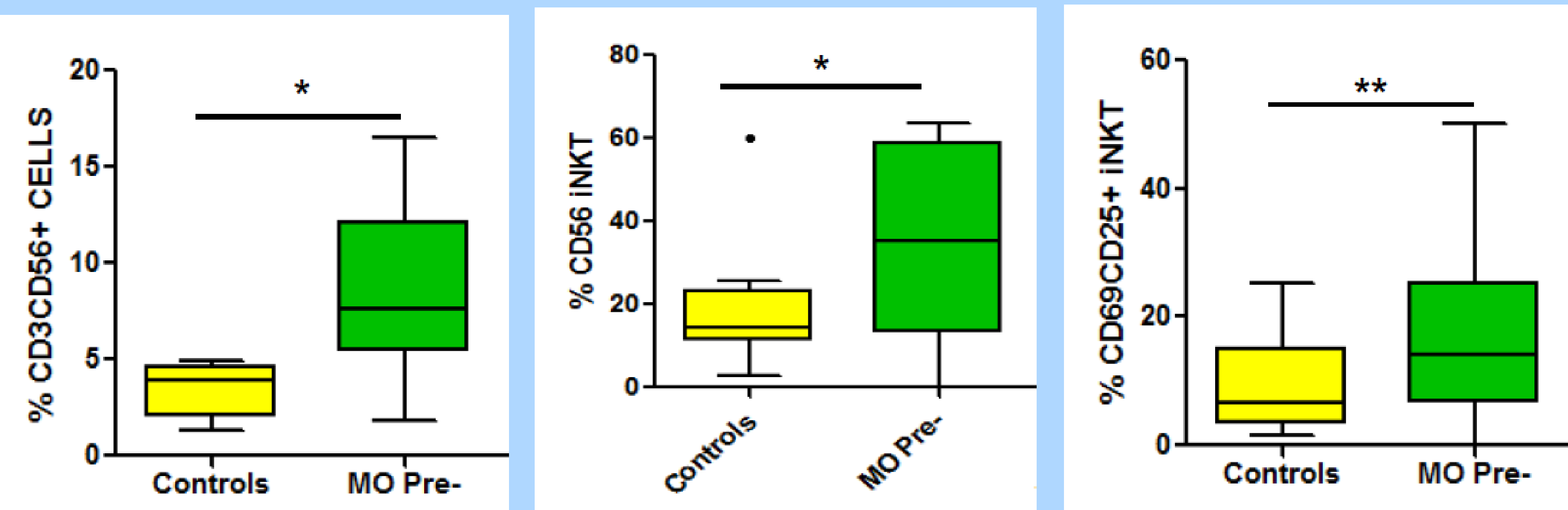

Figure 3. $C D 56+N K T$ and $C D 56+i N K T$ and $C D 69+C D 25+$ iNKT cells were increased in PBMC of MO subjects.

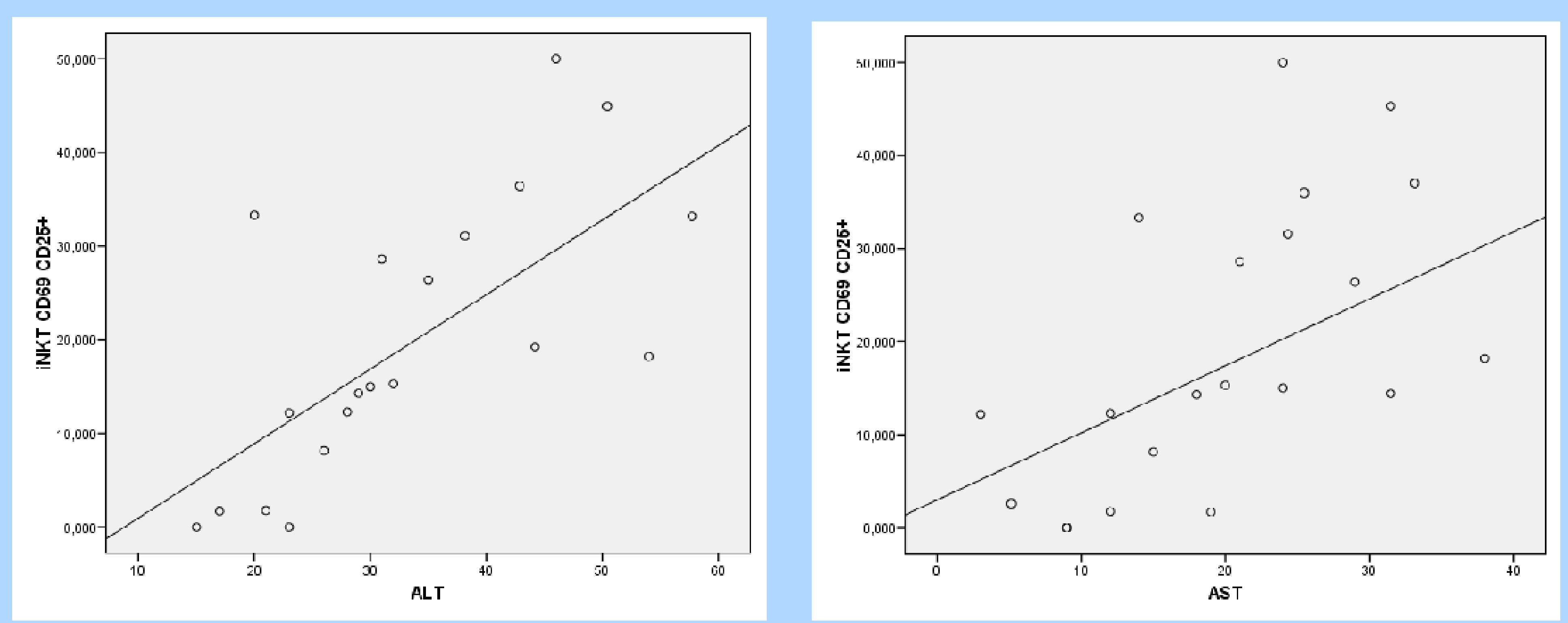

Figure 4. The frequency of CD69+CD25+ iNKT cells (early and later activated iNKT) was significantly and positive correlated with AST and ALT levels.

\section{Conclusions}

MO subjects presented early and later activated lymphocytes and iNKT in PBMC. The direct association found between iNKT cells, both total and activated, in PBMC and serum levels of liver enzymes ALT and AST suggests that this type of cells might play an essential role in fatty liver disease associated to obesity.

This work was supported in part by a grant from the Consejerıa de Economia, Innovacion, Ciencia y Empresa de la Junta de Andalucia, Spain (CTS-8081). This study has been co-funded by FEDER funds.

Email: edugf1@gmail.com

Category: Obesity 